\title{
INEQUALITIES FOR SOME CLASSICAL INTEGRAL TRANSFORMS
}

\author{
PIYUSH KUMAR BHANDARI AND SUSHIL KUMAR BISSU
}

\begin{abstract}
By using a form of the Cauchy-Bunyakovsky-Schwarz inequality, we establish new inequalities for some classical integral transforms such as Laplace transform, Fourier transform, Fourier cosine transform, Fourier sine transform, Mellin transform and Hankel transform.
\end{abstract}

\section{Introduction}

The well-known Cauchy-Bunyakovsky-Schwarz (CBS) inequality [1] states that

$$
\left(\int_{a}^{b} u^{\frac{1}{2}}(x) v^{\frac{1}{2}}(x) d x\right)^{2} \leq\left(\int_{a}^{b} u(x) d x\right)\left(\int_{a}^{b} v(x) d x\right),
$$

for every function $u, v:[a, b] \rightarrow(-\infty, \infty)$ such that the integrals exist.

As we know, the Cauchy Schwarz and Cauchy-Bunyakovsky-Schwarz inequalities play an important role in different branches of modern mathematics such as Hilbert space theory, classical real and complex analysis, numerical analysis, probability and statistics, qualitative theory of differential equations and their applications. To date, a large number of generalizations and refinements of these inequalities have been investigated in the literature (see, e.g., [2]-[8]).

A. Laforgia and P. Natalini [9] used the following form of the CBS inequality (1):

$$
\left(\int_{a}^{b} g(x) f^{\frac{(m+n)}{2}}(x) d x\right)^{2} \leq\left(\int_{a}^{b} g(x) f^{m}(x) d x\right)\left(\int_{a}^{b} g(x) f^{n}(x) d x\right)
$$

to establish some new Turán-type inequalities involving the special functions as gamma, or Polygamma functions and Riemann zeta function. Here $f$ and $g$ are two nonnegative functions of a real variable and $m$ and $n$ belong to a set $S$ of real numbers, such that the involved integrals in (2) exist.

Received July 15, 2015, accepted February 19, 2016. 2010 Mathematics Subject Classification. Primary 26D07; Secondary 44A05.

Key words and phrases. A form of Cauchy-Bunyakovsky-Schwarz inequality, Laplace transform, Fourier transform, convolution theorem, Mellin transform and Hankel transform.

Corresponding author: Piyush Kumar Bhandari. 
Motivated by this remark, we have the idea to replace $u(x)$ and $v(x)$ in (1) by $g^{\gamma}(x) h^{\alpha+\beta}(x)$ and $g^{2-\gamma}(x) h^{\alpha-\beta}(x)$ respectively, to introduce the following new inequality:

$$
\left(\int_{a}^{b} g(x) h^{\alpha}(x) d x\right)^{2} \leq\left(\int_{a}^{b} g^{\gamma}(x) h^{\alpha+\beta}(x) d x\right)\left(\int_{a}^{b} g^{2-\gamma}(x) h^{\alpha-\beta}(x) d x\right)
$$

in which $\alpha, \beta, \gamma \in \mathbb{R}$ and $g, h$ are real integrable functions such that the involved integrals in (3) exist.

The aim of this paper is to apply the continuous inequality (3) for some classical integral transforms in order to get new inequalities.

\section{The Results}

In this section, we apply the inequality (3) to establish new inequalities for some classical integral transforms.

\subsection{An inequality for the Laplace transform}

Theorem 1. Let $L\{f(x) ; p\}$ or $\bar{f}(p)$ be the Laplace transform of a function $f(x)$ defined for $x>0$ with parameter $p$ such that $\operatorname{Re}(p)>0$. Then, for every real numbers $\alpha, \beta$ and $\gamma$ such that $\alpha>0$ and $\alpha>|\beta|$, we have

$$
L^{2}\left\{f\left(\frac{x}{\alpha}\right)\right\} \leq \frac{\alpha^{2}}{\alpha^{2}-\beta^{2}}\left[L\left\{f^{\gamma}\left(\frac{x}{\alpha+\beta}\right)\right\} L\left\{f^{2-\gamma}\left(\frac{x}{\alpha-\beta}\right)\right\}\right] .
$$

Proof. Let $f(x)$ be a function defined for $x>0$, then the Laplace transform [10] of $f(x)$, denoted by $L\{f(x) ; p\}$ or $\bar{f}(p)$, is defined by

$$
L\{f(x) ; p\}=\bar{f}(p)=\int_{0}^{\infty} e^{-p x} f(x) d x
$$

provided that the integral exists for some real or complex value of parameter $p$. In general $\operatorname{Re}(p)>0$, where $\operatorname{Re}(p)$ means real part of $p$.

Now if $g(x)=f(x), h(x)=e^{-p x}$ are substituted in inequality (3) for $[a, b] \rightarrow[0, \infty)$ the following inequality is derived

$$
\begin{aligned}
&\left(\int_{0}^{\infty} e^{-\alpha p x} f(x) d x\right)^{2} \leq\left(\int_{0}^{\infty} e^{-(\alpha+\beta) p x} f^{\gamma}(x) d x\right)\left(\int_{0}^{\infty} e^{-(\alpha-\beta) p x} f^{2-\gamma}(x) d x\right) \\
& \Rightarrow\left(\int_{0}^{\infty} \frac{1}{\alpha} e^{-p u} f\left(\frac{u}{\alpha}\right) d u\right)^{2} \leq\left(\int_{0}^{\infty} \frac{1}{(\alpha+\beta)} e^{-p v} f^{\gamma}\left(\frac{v}{\alpha+\beta}\right) d v\right)\left(\int_{0}^{\infty} \frac{1}{(\alpha-\beta)} e^{-p w} f^{2-\gamma}\left(\frac{w}{\alpha-\beta}\right) d w\right) .
\end{aligned}
$$

By (4), above inequality is equivalent to

$$
\frac{1}{\alpha^{2}} L^{2}\left\{f\left(\frac{x}{\alpha}\right)\right\} \leq \frac{1}{\alpha^{2}-\beta^{2}}\left[L\left\{f^{\gamma}\left(\frac{x}{\alpha+\beta}\right)\right\} L\left\{f^{2-\gamma}\left(\frac{x}{\alpha-\beta}\right)\right\}\right]
$$




$$
\begin{gathered}
\Rightarrow L^{2}\left\{f\left(\frac{x}{\alpha}\right)\right\} \leq \frac{\alpha^{2}}{\alpha^{2}-\beta^{2}}\left[L\left\{f^{\gamma}\left(\frac{x}{\alpha+\beta}\right)\right\} L\left\{f^{2-\gamma}\left(\frac{x}{\alpha-\beta}\right)\right\}\right], \\
\alpha>0, \alpha>|\beta| \text { and } \alpha, \beta, \gamma \in \mathbb{R} .
\end{gathered}
$$

if and only if the corresponding integrals exist.

Corollary 1. Let $L\{f(x) ; p\}$ or $\bar{f}(p)$ be the Laplace transform of a function $f(x)$ defined for $x>0$ with parameter $p$ such that $\operatorname{Re}(p)>0$. Then, for every real numbers $\alpha, \beta$ and $\gamma$ such that $\alpha>0$ and $\alpha>|\beta|$, we have

$$
L^{2}\left\{f\left(\frac{x}{\alpha}\right)\right\} \leq \frac{\alpha^{2}}{\alpha^{2}-\beta^{2}} L\left\{\int_{0}^{x} f^{\gamma}\left(\frac{u}{\alpha+\beta}\right) f^{2-\gamma}\left(\frac{x-u}{\alpha-\beta}\right) d u\right\}
$$

Proof. According to the convolution theorem of Laplace transform, i.e.

$$
L\{f(x)\} L\{g(x)\}=L\left\{\int_{0}^{x} f(u) g(x-u) d u\right\}
$$

Using (6), inequality (5) is in fact equivalent to:

$$
L^{2}\left\{f\left(\frac{x}{\alpha}\right)\right\} \leq \frac{\alpha^{2}}{\alpha^{2}-\beta^{2}} L\left\{\int_{0}^{x} f^{\gamma}\left(\frac{u}{\alpha+\beta}\right) f^{2-\gamma}\left(\frac{x-u}{\alpha-\beta}\right) d u\right\}, \alpha>0, \alpha>|\beta| \text { and } \alpha, \beta, \gamma \in \mathbb{R} .
$$

\subsection{An inequality for the Fourier transform}

Theorem 2. Let $\mathscr{F}\{f(x) ; p\}$ or $\mathscr{F}(p)$ be the Fourier transform of a function $f(x)$ defined on $(-\infty, \infty)$ with parameter $p$. Then, for every real numbers $\alpha, \beta$ and $\gamma$ such that $\alpha>0$ and $\alpha>|\beta|$, we have

$$
\mathscr{F}^{2}\left\{f\left(\frac{x}{\alpha}\right)\right\} \leq \frac{\alpha^{2}}{\alpha^{2}-\beta^{2}}\left[\mathscr{F}\left\{f^{\gamma}\left(\frac{x}{\alpha+\beta}\right)\right\} \mathscr{F}\left\{f^{2-\gamma}\left(\frac{x}{\alpha-\beta}\right)\right\}\right] .
$$

Proof. Let $f(x)$ be a function defined on $(-\infty, \infty)$ and be piecewise-continuously differentiable and absolutely integrable in $(-\infty, \infty)$, then the Fourier transform of $f(x)$, denoted by $\mathscr{F}\{f(x) ; p\}$ or $\mathscr{F}(p)$, is defined as

$$
\mathscr{F}\{f(x) ; p\}=\mathscr{F}(p)=\frac{1}{\sqrt{2 \pi}} \int_{-\infty}^{\infty} e^{i p x} f(x) d x, \quad(-\infty<p<\infty)
$$

Hence, if $g(x)=f(x), h(x)=e^{i p x}$ and $[a, b] \rightarrow(-\infty, \infty)$, are considered in inequality (3) then we get

$$
\begin{aligned}
\left(\int_{-\infty}^{\infty} e^{i \alpha p x} f(x) d x\right)^{2} & \leq\left(\int_{-\infty}^{\infty} e^{i(\alpha+\beta) p x} f^{\gamma}(x) d x\right)\left(\int_{-\infty}^{\infty} e^{i(\alpha-\beta) p x} f^{2-\gamma}(x) d x\right) \\
\Rightarrow\left(\int_{-\infty}^{\infty} \frac{1}{\alpha} e^{i p u} f\left(\frac{u}{\alpha}\right) d u\right)^{2} & \leq\left(\int_{-\infty}^{\infty} \frac{1}{(\alpha+\beta)} e^{i p v} f^{\gamma}\left(\frac{v}{\alpha+\beta}\right) d v\right)\left(\int_{-\infty}^{\infty} \frac{1}{(\alpha-\beta)} e^{i p w} f^{2-\gamma}\left(\frac{w}{\alpha-\beta}\right) d w\right)
\end{aligned}
$$


By (8), above inequality is equivalent to

$$
\begin{aligned}
& \frac{1}{\alpha^{2}} \mathscr{F}^{2}\left\{f\left(\frac{x}{\alpha}\right)\right\} \leq \frac{1}{\alpha^{2}-\beta^{2}}\left[\mathscr{F}\left\{f^{\gamma}\left(\frac{x}{\alpha+\beta}\right)\right\} \mathscr{F}\left\{f^{2-\gamma}\left(\frac{x}{\alpha-\beta}\right)\right\}\right] \\
& \Rightarrow \mathscr{F}^{2}\left\{f\left(\frac{x}{\alpha}\right)\right\} \leq \frac{\alpha^{2}}{\alpha^{2}-\beta^{2}}\left[\mathscr{F}\left\{f^{\gamma}\left(\frac{x}{\alpha+\beta}\right)\right\} \mathscr{F}\left\{f^{2-\gamma}\left(\frac{x}{\alpha-\beta}\right)\right\}\right], \alpha>0, \alpha>|\beta| \text { and } \gamma \in \mathbb{R},
\end{aligned}
$$

if and only if the corresponding integrals exist.

Corollary 2. Let $\mathscr{F}\{f(x) ; p\}$ or $\mathscr{F}(p)$ be the Fourier transform of a function $f(x)$ defined on $(-\infty, \infty)$ with parameter $p$. Then, for every real numbers $\alpha, \beta$ and $\gamma$ such that $\alpha>0$ and $\alpha>|\beta|$, we have

$$
\mathscr{F}^{2}\left\{f\left(\frac{x}{\alpha}\right)\right\} \leq \frac{\alpha^{2}}{\alpha^{2}-\beta^{2}} \frac{1}{\sqrt{2 \pi}} \mathscr{F}\left\{\int_{-\infty}^{\infty} f^{\gamma}\left(\frac{u}{\alpha+\beta}\right) f^{2-\gamma}\left(\frac{x-u}{\alpha-\beta}\right) d u\right\}
$$

Proof. According to the convolution theorem of Fourier transform, i.e.

$$
\mathscr{F}\{f(x)\} \mathscr{F}\{g(x)\}=\mathscr{F}\left\{\frac{1}{\sqrt{2 \pi}} \int_{-\infty}^{\infty} f(u) g(x-u) d u\right\} .
$$

Using (10), inequality (9) is in fact equivalent to:

$$
\mathscr{F}^{2}\left\{f\left(\frac{x}{\alpha}\right)\right\} \leq \frac{\alpha^{2}}{\alpha^{2}-\beta^{2}} \frac{1}{\sqrt{2 \pi}} \mathscr{F}\left\{\int_{-\infty}^{\infty} f^{\gamma}\left(\frac{u}{\alpha+\beta}\right) f^{2-\gamma}\left(\frac{x-u}{\alpha-\beta}\right) d u\right\}, \alpha>0, \alpha>|\beta| \text { and } \gamma \in \mathbb{R} \text {. }
$$

\subsection{An inequality for the Fourier cosine transform}

Theorem 3. Let $\mathscr{F}_{C}\{f(x) ; p\}$ or $\mathscr{F}_{C}(p)$ be the Fourier cosine transform of a function $f(x)$ defined for $x>0$ with parameter $p$ such that $p>0$. Then, for every real numbers $\alpha$ and $\beta$ such that $\alpha \neq \beta$, we have

$$
\mathscr{F}_{C}^{2}\left\{f^{\alpha}(x)\right\} \leq \mathscr{F}_{C}\left\{f^{\alpha+\beta}(x)\right\} \mathscr{F}_{C}\left\{f^{\alpha-\beta}(x)\right\} .
$$

Proof. Let $f(x)$ be a function defined for $x>0$ and be piecewise-continuously differentiable and absolutely integrable in $(0, \infty)$, then the Fourier cosine transform of $f(x)$, denoted by $\mathscr{F}_{C}\{f(x) ; p\}$ or $\mathscr{F}_{C}(p)$, is defined as

$$
\mathscr{F}_{C}\{f(x) ; p\}=\mathscr{F}_{C}(p)=\sqrt{\frac{2}{\pi}} \int_{0}^{\infty} f(x) \cos p x d x, \quad(p>0) .
$$

Hence, if $g(x)=\cos p x, h(x)=f(x),[a, b] \rightarrow[0, \infty)$ and $\gamma=1$ are considered in inequality (3) then we get

$$
\left(\int_{0}^{\infty} f^{\alpha}(x) \cos p x d x\right)^{2} \leq\left(\int_{0}^{\infty} f^{\alpha+\beta}(x) \cos p x d x\right)\left(\int_{0}^{\infty} f^{\alpha-\beta}(x) \cos p x d x\right) .
$$

By applying (12) in above inequality, the following result will eventually be obtained

$$
\mathscr{F}_{C}^{2}\left\{f^{\alpha}(x)\right\} \leq \mathscr{F}_{C}\left\{f^{\alpha+\beta}(x)\right\} \mathscr{F}_{C}\left\{f^{\alpha-\beta}(x)\right\}, \quad \alpha \neq \beta .
$$




\subsection{An inequality for the Fourier sine transform}

Theorem 4. Let $\mathscr{F}_{S}\{f(x) ; p\}$ or $\mathscr{F}_{S}(p)$ be the Fourier sine transform of a function $f(x)$ defined for $x>0$ with parameter $p$ such that $p>0$. Then, for every real numbers $\alpha$ and $\beta$ such that $\alpha \neq \beta$, we have

$$
\mathscr{F}_{S}^{2}\left\{f^{\alpha}(x)\right\} \leq \mathscr{F}_{S}\left\{f^{\alpha+\beta}(x)\right\} \mathscr{F}_{S}\left\{f^{\alpha-\beta}(x)\right\}
$$

Proof. Let $f(x)$ be a function defined for $x>0$ and be piecewise-continuously differentiable and absolutely integrable in $(0, \infty)$, then the Fourier sine transform of $f(x)$, denoted by $\mathscr{F}_{S}\{f(x) ; p\}$ or $\mathscr{F}_{S}(p)$, is defined as

$$
\mathscr{F}_{S}\{f(x) ; p\}=\mathscr{F}_{S}(p)=\sqrt{\frac{2}{\pi}} \int_{0}^{\infty} f(x) \sin p x d x, \quad(p>0) .
$$

Now, applying inequality (3) for $g(x)=\sin p x, h(x)=f(x),[a, b] \rightarrow[0, \infty)$ and $\gamma=1$ results in:

$$
\left(\int_{0}^{\infty} f^{\alpha}(x) \sin p x d x\right)^{2} \leq\left(\int_{0}^{\infty} f^{\alpha+\beta}(x) \sin p x d x\right)\left(\int_{0}^{\infty} f^{\alpha-\beta}(x) \sin p x d x\right) .
$$

By (14), which is equivalent to

$$
\mathscr{F}_{S}^{2}\left\{f^{\alpha}(x)\right\} \leq \mathscr{F}_{S}\left\{f^{\alpha+\beta}(x)\right\} \mathscr{F}_{S}\left\{f^{\alpha-\beta}(x)\right\}, \quad \alpha \neq \beta .
$$

\subsection{An inequality for the Mellin transform}

Theorem 5. Let $M\{f(x) ; p\}$ or $M(p)$ be the Mellin transform of a function $f(x)$ defined for $x>0$ with parameter $p$. Then, for every real numbers $\alpha$ and $\beta$ such that $\alpha \neq \beta$, we have

$$
M^{2}\left\{f^{\alpha}(x)\right\} \leq M\left\{f^{\alpha+\beta}(x)\right\} M\left\{f^{\alpha-\beta}(x)\right\} .
$$

Proof. Let a function $f(x)$ be defined for $0<x<\infty$, then the Mellin transform of $f(x)$, denoted by $M\{f(x) ; p\}$ or $M(p)$, is defined as

$$
M\{f(x) ; p\}=M(p)=\int_{0}^{\infty} x^{p-1} f(x) d x .
$$

Hence, if $g(x)=x^{p-1}, h(x)=f(x),[a, b] \rightarrow[0, \infty)$ and $\gamma=1$ are considered in inequality (3), then we get

$$
\left(\int_{0}^{\infty} x^{p-1} f^{\alpha}(x) d x\right)^{2} \leq\left(\int_{0}^{\infty} x^{p-1} f^{\alpha+\beta}(x) d x\right)\left(\int_{0}^{\infty} x^{p-1} f^{\alpha-\beta}(x) d x\right) .
$$

By (16), this can be written as

$$
M^{2}\left\{f^{\alpha}(x)\right\} \leq M\left\{f^{\alpha+\beta}(x)\right\} M\left\{f^{\alpha-\beta}(x)\right\} \quad \alpha \neq \beta .
$$

This completes the proof. 


\subsection{An inequality for the Hankel transform}

Theorem 6. Let $H_{v}\{f(x) ; p\}$ or $H_{v}(p)$ be the Hankel transform of a function $f(x)$ defined for $x>0$ with order $v \geq 0$ and parameter $p$. Then, for every real numbers $\alpha$ and $\beta$ such that $\alpha \neq \beta$, we have

$$
H_{v}^{2}\left\{f^{\alpha}(x)\right\} \leq H_{v}\left\{f^{\alpha+\beta}(x)\right\} H_{v}\left\{f^{\alpha-\beta}(x)\right\} .
$$

Proof. Let $f(x)$ be a function defined for all positive values of the variable $x$, i.e., for $x>0$, Then the Hankel transform of $f(x)$, denoted by $H_{v}\{f(x) ; p\}$ or $H_{v}(p)$, is defined as

$$
H_{v}\{f(x) ; p\}=H_{v}(p)=\int_{0}^{\infty} x J_{v}(p x) f(x) d x
$$

where $J_{v}(p x)$ is the Bessel function of the first kind of order $v \geq 0$.

By (3) with $g(x)=x J_{v}(p x), h(x)=f(x),[a, b] \rightarrow[0, \infty)$ and $\gamma=1$, we get

$$
\left(\int_{0}^{\infty} x J_{v}(p x) f^{\alpha}(x) d x\right)^{2} \leq\left(\int_{0}^{\infty} x J_{v}(p x) f^{\alpha+\beta}(x) d x\right)\left(\int_{0}^{\infty} x J_{v}(p x) f^{\alpha-\beta}(x) d x\right) .
$$

By (18), this is equivalent to

$$
H_{v}^{2}\left\{f^{\alpha}(x)\right\} \leq H_{v}\left\{f^{\alpha+\beta}(x)\right\} H_{v}\left\{f^{\alpha-\beta}(x)\right\} \quad \alpha \neq \beta .
$$

This completes the proof.

\section{References}

[1] D. S. Mitrinovic, J. E. Pecaric and A. M. Fink, Classical and New Inequalities in Analysis, Kluwer Academic, Dordrecht, 1993.

[2] D. K. Callebaut, Generalization of the Cauchy-Schwartz inequality, J. Math. Anal. Appl., 12 (1965), $491-494$.

[3] W. L. Steiger, On a generalization of the Cauchy-Schwarz inequality, Amer. Math. Monthly, 76 (1969), $815-$ 816.

[4] L. Zheng, Remark on a refinement of the Cauchy-Schwartz inequality, J. Math. Anal. Appl., 218 (1998), 13-21.

[5] H. Alzer, On the Cauchy-Schwartz inequality, J. Math. Anal. Appl., 234 (1999), 6-14.

[6] M. Masjed-Jamei, S. S. Dragomir and H. M. Srivastava, Some generalizations of the Cauchy-Schwarz and the Cauchy-Bunyakovsky inequalities involving four free parameters and their applications, Math. Computer Modeling, 49 (2009), 1960-1968.

[7] Cristinel Mortici, New Inequalities for some special functions via the Cauchy-Bunyakovsky-Schwarz inequality, Tamkang Journal of Mathematics, 42 (2011), 53-57.

[8] M. Masjed-Jamei, A functional generalization of the Cauchy-Schwarz inequality and some subclasses, Appl. Math. Lett., 22 (2009), 1335-1339.

[9] A. Laforgia and P. Natalini, Turán-type inequalities for some special functions, J. Inequal. Pure Appl. Math., 27 (2006), Issue 1, Art. 32.

[10] M. Abramowitz and I. A. Stegun (Eds.), Handbook of Mathematical Functions with Formulas, Graphs and Mathematical Tables, Dover Publications, Inc., New York, 1965.

Department of Mathematics, Shrinathji Institute of Technology \& Engineering, Nathdwara, Rajasthan 313301, India.

E-mail: bhandari1piyush@gmail.com

Department of Mathematics, Government College of Ajmer, Ajmer, Rajasthan 305004, India.

E-mail: susilkbissu@gmail.com 